\title{
Impact of concomitant systemic treatments on toxicity and intracerebral response after stereotactic radiotherapy for brain metastases
}

Morgan Guénolé ${ }^{1}$, François Lucia ${ }^{1,2^{*}}$ D, Vincent Bourbonne ${ }^{1,2}$, Gurvan Dissaux ${ }^{1,2}, E^{2}$ manuelle Reygagne ${ }^{1}$, Gaëlle Goasduff', Olivier Pradier ${ }^{1,2}$ and Ulrike Schick ${ }^{1,2}$

\begin{abstract}
Background: The aim of this study was to determine the safety and efficacy of fractionated stereotactic radiotherapy (SRT) in combination with systemic therapies (ST) for brain metastases (BM).

Methods: Ninety-nine patients (171 BM) received SRT and concurrent ST (group 1) and 95 patients (131 BM) received SRT alone without concurrent ST (group 2). SRT was planned on a linear accelerator, using volumetric modulated arc therapy. All ST were allowed including chemotherapy (CT), immunotherapy (IT), targeted therapy (TT) and hormonotherapy $(\mathrm{HT})$. Treatment was considered to be concurrent if the timing between the drug administration and SRT did not exceed 1 month. Local control (LC), freedom for distant brain metastases (FFDBM), overall survival (OS) and radionecrosis (RN) were evaluated.

Results: After a median follow-up of 11.9 months (range 0.7-29.7), there was no significant difference between the two groups. However, patients who received concurrent IT $(n=30)$ had better 1-year LC, OS, FFDBM but a higher RN rate compared to patients who did not: $96 \%$ versus $78 \%(p=0.02), 89 \%$ versus $77 \%(p=0.02), 76 \%$ versus $53 \%$ $(p=0.004)$ and $80 \%$ versus $90 \%(p=0.03)$, respectively. In multivariate analysis, concurrent IT $(p=0.022)$ and tumor volume $<2.07 \mathrm{CC}(p=0.039)$ were significantly correlated with improvement of LC. The addition of IT to SRT compared to SRT alone was associated with an increased risk of RN $(p=0.03)$.
\end{abstract}

Conclusion: SRT delivered concurrently with IT seems to be associated with improved LC, FFDBM and OS as well as with a higher rate of RN.

Keywords: Stereotactic radiotherapy, Brain metastases, Systemic therapies, Immunotherapy, Radioimmunotherapy

\section{Background}

Brain metastases (BM) are frequent in the natural history of several solid tumors and represent an important cause of morbidity and mortality despite active treatments [1]. For several decades, palliative whole

\footnotetext{
* Correspondence: francois.lucia@gmail.com

'Radiation Oncology Department, University Hospital Morvan, 2 Avenue Foch, F-29200 Brest, France

²Latim INSERM UMR 1101, UBO, Brest, France
}

brain radiotherapy (RT) has been the standard of care in these patients, allowing however only minor improvement in overall survival (OS) at the price of substantial neurotoxicity [2-4]. In the last decade, stereotactic radiotherapy (SRT) has emerged as a local and potentially curative treatment in a subset of patients with a limited number of BM. This approach indeed improves local control rates and reduces toxicity compared to whole brain RT [5-7].

(c) The Author(s). 2020 Open Access This article is licensed under a Creative Commons Attribution 4.0 International License, which permits use, sharing, adaptation, distribution and reproduction in any medium or format, as long as you give appropriate credit to the original author(s) and the source, provide a link to the Creative Commons licence, and indicate if changes were made. The images or other third party material in this article are included in the article's Creative Commons licence, unless indicated otherwise in a credit line to the material. If material is not included in the article's Creative Commons licence and your intended use is not permitted by statutory regulation or exceeds the permitted use, you will need to obtain permission directly from the copyright holder. To view a copy of this licence, visit http://creativecommons.org/licenses/by/4.0/ The Creative Commons Public Domain Dedication waiver (http://creativecommons.org/publicdomain/zero/1.0/) applies to the data made available in this article, unless otherwise stated in a credit line to the data. 
For a long time, double-strand DNA damage in tumor cells and microenvironment were considered as the predominant mechanisms of action of RT [8]. But, recently, several studies have shown that RT, in response to antigen presentation, can induce immunomodulatory changes such as release of cytokines, increase in tumorinfiltrating lymphocytes and destruction of immunosuppressive stromal cells, allowing enhancement of immunogenic cell death $[9,10]$.

The wide implementation of SRT for BM management occurs in the era of new systemic treatment options such as tyrosine kinase inhibitors (TKI) and checkpoint inhibitors. By enhancing the host's immune response against tumor cells, immune checkpoint inhibitors such as anti programmed death-1 (PD-1)/anti programmed death ligand-1 (PD-L1) and anti-cytotoxic $\mathrm{T}$ lymphocyte-associated antigen 4 (CTLA-4) monoclonal antibodies have revolutionized the treatment of multiple cancers including melanoma, non-small cell lung cancer and renal carcinoma [11-13]. Nevertheless, only a minority of patients achieve complete response. Additional strategies are thus necessary to improve treatment efficacy. As these drugs increase tumor cells sensitivity to radiation, the synergy between these modalities represents a new therapeutic option $[14,15]$.

Retrospective studies have shown SRT to be safe in combination with systemic therapies, especially in melanoma [16-18]. However, concerns exist regarding the potential toxicity given the interaction complexity between RT and systemic therapies and there is still an outstanding need for safety data on these therapeutic associations.

The aim of this study was to determine the safety and efficacy of SRT in combination with systemic therapies for intracranial metastases.

\section{Methods}

\section{Patients characteristics}

Patients treated with SRT for BM between 12/2014 and $06 / 2018$ at our institution were included in this study. All primary tumors histologies were considered. Systemic treatments and SRT were suggested at multidisciplinary team meetings. Patients were offered SRT if the following criteria were met: number of $\mathrm{BM} \leq 6$, larger diameter $<40 \mathrm{~mm}$ according to magnetic resonance imaging (MRI), life expectancy $>3$ months according to Diagnosis-Specific Graded Prognostic Assessment (DSGPA), and non critical anatomic position. In patients who underwent SRT treatment more than once, each new lesion was considered separately.

The diagnosis was done by the MRI examination included Fluid-attenuated inversion recovery (FLAIR) and T1 weighted sequences. Extracranial disease status was defined by thoraco-abdomino-pelvic computed tomography $(\mathrm{CT})$ with contrast enhancement or $18 \mathrm{~F}$ fluorodeoxyglucose (FDG) positron emission tomography/computed tomography (PET/CT).

All systemic treatments were allowed in this study including chemotherapy, immunotherapy, targeted therapy and hormonotherapy. Nivolumab was administrated at a dose of $3 \mathrm{mg} / \mathrm{kg}$ every 2 weeks, pembrolizumab at a dose of $2 \mathrm{mg} / \mathrm{kg}$ every 3 weeks, durvalumab at a dose $10 \mathrm{mg} /$ $\mathrm{kg}$ every 2 weeks and ipilimumab at a dose of $3 \mathrm{mg} / \mathrm{kg}$ every 3 weeks, by intravenous injection. Targeted therapies were mainly used in non small cell lung cancers (anaplastic lymphoma kinase (ALK) inhibitor) and in renal cancer (TKI). More than 20 different chemotherapy regimens were used depending on the primary tumor and disease course. Platinum salts and taxane were the most represented CT drugs in patients with lung and breast cancer, respectively.

Treatment was considered to be concurrent if the timing between the drug administration and SRT did not exceed 1 month $[19,20]$. If systemic treatment was administered before SRT, the date of SRT start was considered. On the opposite, in patients who received systemic treatment after irradiation, the date of the last SRT fraction was considered.

The cohort was divided into 2 groups: group 1 included patients treated with SRT and concurrent systemic treatment and group 2 included patients treated with SRT alone. All patients provided signed permission for the use of their clinical data for scientific purposes and Institutional Review Board approved this study.

\section{Treatment planning}

According to different clinical and radiological parameters including BM size, presence of acute neurological symptoms, proximity to organs at risk (OAR), and critical anatomical position, total dose was prescribed at the planning target volume (PTV) periphery and ranged between 21 and $23.1 \mathrm{~Gy}$ in 3 fractions. Treatment plans were generated for a TrueBeamTM STX linac (Varian Medical Systems, Palo Alto, CA) equipped with standard Millennium MultiLeaf Collimator (MLC) with 120 leaves (thickness of $2.5 \mathrm{~mm}$ at isocenter and up to $8 \mathrm{~cm}$ followed by a thickness of $5 \mathrm{~mm}$ from 8 to $22 \mathrm{~cm}$ ). Every patient was planned using a Flattening-Filter (FF) volumetric modulated arctherapy (VMAT) technique with 6X beams in the Pinnacle ${ }^{\oplus}$ treatment planning system (Philips, v9.10). We used two arcs from $182^{\circ}$ to $178^{\circ}$ for each VMAT plan. The maximum dose rate was set to $600 \mathrm{MU} / \mathrm{min}$ for $6 \mathrm{X}$ beams.

For patients treated until 06/2017, the prescribed dose to the PTV was a uniform dose of $3 \times 7.7 \mathrm{~Gy}$ in the periphery of the PTV. In the following patients, the planning protocol was modified and a dose gradient was created inside the PTV: the dose prescribed was $3 \times 11$ 
Gy at the isocenter with the $70 \%$ isodose line covering $99 \%$ of the PTV.

\section{Tumor response assessment and toxicity}

Patients were assessed by brain MRI and neurological examination 6 weeks after SRT completion and every 3 months thereafter.

Radiological response on MRI was assessed by an expert neuroradiologist according to the Response Assessment for BM (RANO-BM) criteria [21]: local control (LC) was defined as the absence of new enhancing abnormality in the irradiated areas, and distant brain metastasis (DBM) as the presence of new BM or leptomeningeal enhancement outside the treated region.

Radionecrosis (RN) was assessed using contrast enhanced T1-, T2 weighted- and perfusion-MRI, even in asymptomatic patients. It was considered as the presence of central hypo-intensity and peripheral enhancement on T1-weighted post-contrast imaging, associated with edema on T2-weighted sequences and a lack of perfusion without any nodular highly vascularized area within the contrast enhanced lesion on perfusion MRI. If multiparametric MRI was inconclusive, an F-18 fluoroethyltyrosine (FET)-PET was performed. Histological confirmation of RN was not systematically required.

Systemic disease was evaluated by contrast enhanced total body CT scan, and/or 18-FDG CT-PET, depending on the primary disease.

\section{Statistical analysis}

The general data behavior was described by the used of standard descriptive statistics. LC was defined from the SRT start to time of the local relapse. Intracranial progression was considered from the SRT start to the time of any new central nervous system progression. Overall survival (OS) was calculated from the date of diagnosis to the death or last follow-up date. Cut-off values of significant parameters (age, DS-GPA, RPA, number of BM, KPS, GTV volume and size of BM) were calculated using the receiver operating characteristic (ROC).

Univariate and multivariate Cox model was used as a method to estimate the independent association of a variable set with $\mathrm{LC}$, freedom from distant brain metastases (FFDBM) and RN. All statistical analyses were performed using MedCalc Statistical Software version 15.8 (MedCalc Software bvba, Ostend, Belgium).

\section{Results}

Patient characteristics, radiation and systemic treatments A total of 194 patients treated with SRT for 302 BM were included in this study: the first group (patients treated with SRT and concurrent systemic treatments) included 99 patients with a total of $171 \mathrm{BM}$. The second group (patients with SRT alone, without concurrent treatment) included 95 patients with a total of $131 \mathrm{BM}$.

Median age was 60 years. The majority of patients were male (56\%), were in RPA class II (64\%), had a KPS of $90-100 \%$ (70\%), a DS-GPA score of $2.5-3$ (53\%), and a primary lung cancer $(71.1 \%)$. Whole brain RT had been performed prior to SRT in $25 \%$ of patients.

Both groups were similar in terms of SRT parameters, clinical and histopathological characteristics except regarding the primary tumor (lung cancer being more represented in group 2), RPA class and the presence of extra cerebral metastases (more represented in group 1) (Table 1).

Regarding systemic therapy, more patients were treated with IT in group $1(p<0.001)$ and with CT in group $2(p<0.001)$. The median SRT dose was 23.1Gy in 3 fractions, with an inhomogenous dose prescription in $49 \%$ of patients $(150 / 302 \mathrm{BM})$ (Table 1$)$.

\section{Local control, distant brain free metastases and overall survival analysis}

Median follow-up time was 11.6 months (1.4-17.3) in the first group and 12.1 months in the second group $(0.7-29.7)$.

\section{Local control}

Local failures occurred in 52/302 (17\%) irradiated metastases. LC at 6-months and 1-year were 93\% (IC95\% [9196]) and $87 \%$ (IC95\% [83-91]), respectively. Twentyseven out of 171 (15.7\%) and 25/131 (19.1\%) BM were not locally controlled in group 1 and 2 , respectively ( $p=$ $0.3)$. On univariate analysis, factors recorded as influencing positively LC were tumor volume $(<2.07 \mathrm{cc})$, age $(<$ 55 years), DS-GPA score ( $>2.5$ ), prior WBRT and concurrent IT. On multivariate analysis, two factors remained statistically associated with LC: tumor volume $(p=0.039)$ and concomitant IT $(p=0.022)$ (Table 2). The estimated 1-year LC rates were 96 and $78 \%$ in BM irradiated with versus without concomitant IT $(p=0.02)$ (figure 1A, supplementary materials). However, there was no impact of the treatments sequences (IT before or after RT). Similarly, 90 and $72 \%$ BM with low versus high tumor volume (cut off $2.07 \mathrm{cc}$ ) were still controlled at 1 year $(p=0.03)$ (figure $1 \mathrm{~B}$, supplementary materials).

\section{Distant brain free metastases survival}

Brain distance failures occurred in 60/194 patients (30.9\%): $27 / 99$ patients (27.3\%) in group 1 and 33/95 patients $(34.7 \%)$ in group $2(p=0.3)$. The 1 -year freedom for distant brain metastases (FFDBM) rate was 58\% (IC95\% [53.8-60.6]). On univariate analysis, factors influencing FFDBM were number of BM (>1), dose distribution (homogeneous vs inhomogeneous), concurrent systemic treatment, IT administration, and 
Table 1 Patients' characteristics

\begin{tabular}{|c|c|c|c|c|c|}
\hline & $\begin{array}{l}\text { Groupe } 1 \\
\text { Concomitant } \\
N=99 \text { (171 metastases) }\end{array}$ & $\%$ & $\begin{array}{l}\text { Group } 2 \\
\text { Non concomitant } \\
N=95 \text { (131 metastases) }\end{array}$ & $\%$ & Difference $p$-value \\
\hline Gender & & & & & 0.124 \\
\hline Female & 49 & 49 & 36 & 38 & \\
\hline Male & 50 & 51 & 59 & 62 & \\
\hline KPS median (range) & 90 & $(60-100)$ & 90 & $(60-100)$ & 0.230 \\
\hline Age median (range) & 61 & $(38-85)$ & 60 & $(24-84)$ & 0.530 \\
\hline \multicolumn{6}{|l|}{ Primary } \\
\hline Lung & $60 / 99$ & 60 & $70 / 95$ & 75 & 0.004 \\
\hline EGFR+ & $18 / 60$ & 30 & $1 / 70$ & 1.5 & \\
\hline ALK+ & $7 / 60$ & 12 & $3 / 70$ & 4 & \\
\hline PDL1 > 1\% & $26 / 60$ & 43 & $1 / 70$ & 1.5 & \\
\hline Breast & 19/99 & 19 & $9 / 95$ & 9 & 0.177 \\
\hline HER2+++ & 13/19 & 68 & $1 / 9$ & 11 & \\
\hline Melanoma & $13 / 99$ & 13 & $4 / 95$ & 4 & 0.049 \\
\hline GU & $2 / 99$ & 2 & $4 / 95$ & 4 & 0.987 \\
\hline $\mathrm{Gl}$ & $5 / 99$ & 5 & $8 / 95$ & 8 & 0.461 \\
\hline Number of BMs (range) & 2 & $(1-5)$ & 2 & $(1-7)$ & 0.998 \\
\hline \multicolumn{6}{|l|}{ Tumor size $(\mathrm{cm})$} \\
\hline$<2 \mathrm{~cm}$ & 129/171 & 76 & $88 / 131$ & 67 & 0.109 \\
\hline $2-3 \mathrm{~cm}$ & $28 / 171$ & 16 & $32 / 131$ & 25 & 0.072 \\
\hline$>3 \mathrm{~cm}$ & $14 / 171$ & 8 & $11 / 131$ & 8 & 0.831 \\
\hline \multicolumn{6}{|l|}{ Prior WBRT } \\
\hline Yes & 25 & 25 & 24 & 26 & 0.995 \\
\hline No & 74 & 74 & 71 & 74 & \\
\hline DS GPA (range) & 2,5 & $(0.5-3.5)$ & 2,5 & $(0.5-3.5)$ & 0.999 \\
\hline \multicolumn{6}{|l|}{ RPA } \\
\hline 1 & 18/99 & 18 & $45 / 95$ & 47 & $<0.001$ \\
\hline$\|$ & 78/99 & 79 & $47 / 95$ & 50 & $<0.001$ \\
\hline III & $3 / 99$ & 3 & $3 / 95$ & 3 & 0.673 \\
\hline \multicolumn{6}{|l|}{ ECM } \\
\hline Yes & 56 & 56 & 32 & 34 & 0.002 \\
\hline No & 43 & 43 & 63 & 66 & \\
\hline \multicolumn{6}{|l|}{ Treatments } \\
\hline$C T$ & $33 / 99$ & 33 & $64 / 95$ & 67 & $<0.001$ \\
\hline Before & $14 / 33$ & & $48 / 64$ & & \\
\hline After & $23 / 33$ & & $19 / 64$ & & \\
\hline $\mathrm{IT}$ & $30 / 99$ & 30 & $6 / 95$ & 6 & $<0.001$ \\
\hline Before & $15 / 30$ & & $4 / 6$ & & \\
\hline After & $16 / 30$ & & $2 / 6$ & & \\
\hline$\pi$ & $36 / 99$ & 36 & $17 / 95$ & 18 & 0.031 \\
\hline Before & $22 / 36$ & & $9 / 17$ & & \\
\hline After & $27 / 33$ & & $8 / 17$ & & \\
\hline $\mathrm{HT}$ & $4 / 99$ & 4 & $1 / 94$ & 1 & 0.403 \\
\hline
\end{tabular}

Dosimetric parameters 
Table 1 Patients' characteristics (Continued)

\begin{tabular}{|c|c|c|c|c|c|}
\hline & $\begin{array}{l}\text { Groupe } 1 \\
\text { Concomitant } \\
N=99 \text { (171 metastases) }\end{array}$ & $\%$ & $\begin{array}{l}\text { Group } 2 \\
\text { Non concomitant } \\
N=95 \text { (131 metastases) }\end{array}$ & $\%$ & Difference $p$-value \\
\hline HG & $87 / 171$ & 51 & $65 / 131$ & 49 & 0.783 \\
\hline $\mathrm{INH}$ & $84 / 171$ & 49 & $66 / 131$ & 51 & \\
\hline
\end{tabular}

Abbreviations: KPS Karnofsky Performance Status, EGFR Epidermal Growth Factor Receptor, ALK Anaplastic lymphoma kinase, PLD1 Programmed death-ligand 1, HER2 Human Epidermal Growth Factor Receptor-2, GU Genito-urinary, GI Gastro-Intestinal, BM Brain metastases, WBRT Whole brain radiotherapy, DS-GPA Diagnostic-Specific Graded Prognostic Assessment, RPA Recursive partitioning analysis, ECM Extracranial metastases, CT Chemotherapy, IT Immunotherapy, $\Pi T$ Targeted therapy, HT Hormonotherapy, HG Homogeneous, INH Inhomogeneous

concurrent IT. There was no impact of $\mathrm{TT}$ or CT ( $p=0.78$ and 0.82 respectively) on FFDBM, even when combined with SRT. On multivariate analysis, 3 factors remained statistically associated with FFDBM (Table 3): number of BMs $(p<0.001)$, concomitant systemic treatment $(p<0.001)$ and dose distribution $(p<0.001)$. Patients who did and did not receive concurrent systemic treatment had a 1-year FFDBM rate of 62 and 51\%, respectively $(p=0.007)$. The estimated 1-year FFDBM rates with homogeneous versus inhomogeneous dose prescription were 47 and $71 \%$ $(p<0.001)$ (figure 2A, supplementary materials). Regarding the possible impact of concurrent IT, the 1-year FFDBM rate with concurrent IT versus no concurrent IT were 76 and $53 \%$ respectively ( $p=$
0.004) (figure 2B, supplementary materials). This parameter tends to significance in multivariate analysis $(p=0.08)$.

\section{Overall survival}

At the last follow-up, 120/194 patients were alive (62\%): $66 / 99$ patients $(66.7 \%)$ in group 1 and 54/95 patients (56.8) in group $2(p=0.2)$. The overall 1 -year OS rate was $80 \%$ (IC95\% [77.6-82.9]). On univariate analysis, age $\leq 55$ years, DS-GPA $>2.5$, RPA class $=1$, absence of extra-cranial metastasis, Karnosky performance status (KPS) $\geq 80 \%$, tumor volume $<2.07 \mathrm{cc}$ and concurrent IT were significantly correlated with improved survival. However, when considering concurrent systemic treatment as a whole, no impact on OS was noted. On

Table 2 Univariate and multivariate analysis for local control (LC)

\begin{tabular}{|c|c|c|c|c|c|c|}
\hline \multirow[t]{2}{*}{ Variables } & \multirow[t]{2}{*}{ HR } & \multicolumn{2}{|c|}{ Univariate analysis } & \multirow[t]{2}{*}{ OR } & \multicolumn{2}{|c|}{ Multivariate analysis } \\
\hline & & IC95\% & $p$ & & IC95\% & $p$ \\
\hline Histology (lung vs. others) & 0.84 & $0.47-1.51$ & 0.57 & & & \\
\hline Age $(\leq 55$ vs. $>55)$ & 1.94 & $2.06-3.58$ & 0.01 & 0.98 & $0.95-1.01$ & 0.11 \\
\hline Gender (male vs. female) & 1.29 & $0.75-2.22$ & 0.35 & & & \\
\hline DS-GPA (> 2.5 vs. $\leq 2.5)$ & 0.49 & $0.27-0.88$ & 0.02 & 0.87 & $0.79-1.87$ & 0.39 \\
\hline RPA (1 vs. $>1)$ & 0.73 & $0.41-1.28$ & 0.29 & & & \\
\hline Number of BM (1 vs. > 1) & 0.69 & $0.39-1.23$ & 0.23 & & & \\
\hline ECM (yes vs. No) & 1.01 & $0.57-1.76$ & 0.84 & & & \\
\hline KPS (< $80 \%$ vs. $\geq 80 \%)$ & 1.40 & $0.74-2.62$ & 0.29 & & & \\
\hline GTV volume $(\geq 2.07$ vs. $<2.07)$ & 1.75 & $1.00-3.06$ & 0.03 & 2.04 & $1.03-4.01$ & 0.039 \\
\hline Size (> $10 \mathrm{~mm}$ vs. $<10 \mathrm{~mm}$ ) & 1.16 & $0.64-2.10$ & 0.61 & & & \\
\hline Dose distribution (HG vs. INH) & 0.58 & $0.34-1.01$ & 0.06 & & & \\
\hline ST concurrently (group 1 vs. group 2) & 0.73 & $0.42-1.28$ & 0.32 & & & \\
\hline IT (yes vs. no) & 0.68 & $0.35-1.31$ & 0.30 & & & \\
\hline IT concurrently (yes vs. no IT) & 0.33 & $0.16-0.66$ & 0.02 & 0.18 & $0.12-0.79$ & 0.022 \\
\hline TT (yes vs. no) & 0.93 & $0.52-1.66$ & 0.93 & & & \\
\hline TT concurrently (yes vs. no TKI) & 0.92 & $0.50-1.67$ & 0.79 & & & \\
\hline CT (yes vs. no) & 1.22 & $0.71-2.09$ & 0.46 & & & \\
\hline $\mathrm{CT}$ concurrently (yes vs. no $\mathrm{CT}$ ) & 0.79 & $0.46-1.37$ & 0.40 & & & \\
\hline Prior WBRT (yes vs. no) & 2.26 & $1.16-4.48$ & 0.003 & 0.93 & $0.83-2.62$ & 0.14 \\
\hline
\end{tabular}

Abbreviations: DS-GPA Diagnostic-Specific Graded Prognostic Assessment, RPA Recursive partitioning analysis, BM Brain metastases, ECM Extracranial metastases, KPS Karnofsky Performance Status, GTV Gross Tumor Volume, HG Homogeneous, INH Inhomogeneous, ST Systemic Treatment, IT Immunotherapy, $T T$ Targeted therapy, CT Chemotherapy, WBRT Whole brain radiotherapy 
Table 3 Univariate and multivariate analysis for freedom for distant brain metastases

\begin{tabular}{|c|c|c|c|c|c|c|}
\hline \multirow[t]{2}{*}{ Variables } & \multirow[t]{2}{*}{ HR } & \multicolumn{2}{|c|}{ Univariate analysis } & \multirow[t]{2}{*}{ OR } & \multicolumn{2}{|c|}{ Multivariate analysis } \\
\hline & & IC95\% & $p$ & & IC95\% & $p$ \\
\hline Histology (lung vs. others) & 0.90 & $0.56-1.38$ & 0.62 & & & \\
\hline Age (> 55 vs. $\leq 55$ ) & 1.19 & $0.77-1.86$ & 0.39 & & & \\
\hline Gender (male vs. female) & 1.19 & $0.80-1.77$ & 0.38 & & & \\
\hline DS-GPA (> 2.5 vs. $\leq 2.5)$ & 0.90 & $0.61-1.34$ & 0.29 & & & \\
\hline RPA (1 vs. > 1) & 0.86 & $0.57-1.29$ & 0.45 & & & \\
\hline Number of BM (> 1 vs. 1 ) & 1.52 & $1.03-2.54$ & 0.03 & 1.42 & $1.18-1.72$ & $<0.001$ \\
\hline ECM (yes vs. No) & 0.99 & $0.67-1.48$ & 0.99 & & & \\
\hline KPS (<80\% vs. $\geq 80 \%)$ & 1.18 & $0.77-1.80$ & 0.35 & & & \\
\hline GTV volume $(<2.07$ vs. $\geq 2.07)$ & 0.81 & $0.55-1.80$ & 0.25 & & & \\
\hline Size (> $10 \mathrm{~mm}$ vs. $<10 \mathrm{~mm}$ ) & 1.12 & $0.73-1.70$ & 0.58 & & & \\
\hline Dose distribution (HG vs. INH) & 2.09 & $1.42-3.06$ & $<0.001$ & 1.53 & $1.36-1.93$ & $<0.001$ \\
\hline ST concurrently (group 1 vs. group 2) & 0.60 & $0.40-0.88$ & 0.007 & 0.37 & $0.21-0.67$ & $<0.001$ \\
\hline IT (yes vs. no) & 0.47 & $0.29-0.75$ & 0.001 & 0.93 & $0.75-4.36$ & 0.70 \\
\hline IT concurrently (yes vs. no IT) & 0.38 & $0.24-0.63$ & 0.004 & 0.77 & $0.68-1.12$ & 0.08 \\
\hline TT (yes vs. no) & 0.81 & $0.54-1.21$ & 0.32 & & & \\
\hline TT concurrently (yes vs. no TKI) & 1.07 & $0.70-1.92$ & 0.78 & & & \\
\hline CT (yes vs. no) & 1.06 & $0.72-1.55$ & 0.76 & & & \\
\hline $\mathrm{CT}$ concurrently (yes vs. no CT) & 0.96 & $0.65-1.41$ & 0.82 & & & \\
\hline Prior WBRT (yes vs. no) & 0.68 & $0.43-1.08$ & 0.07 & & & \\
\hline
\end{tabular}

Abbreviations: DS-GPA Diagnostic-Specific Graded Prognostic Assessment, RPA Recursive partitioning analysis, BM Brain metastases, ECM Extracranial metastases, KPS Karnofsky Performance Status, GTV Gross Tumor Volume, HG Homogeneous, INH Inhomogeneous, ST Systemic treatment, IT Immunotherapy, $T T$ Targeted therapy, CT Chemotherapy, WBRT Whole brain radiotherapy

multivariate analysis, age, RPA score, KPS and concurrent IT remained statistically associated with improvement of OS (Table 4). The estimated 1-year OS rate was $89 \%$ versus $77 \%$ in patients treated with concurrent IT compared to those who were not $(p=0.02)$ (Fig. 1).

\section{Toxicity}

SRT was well tolerated, and few acute RT-related adverse events occurred: Grade 1 and 2 nausea and headaches were reported in 17 patients (9\%) with no significant difference between the 2 groups $(10 \%$ in group 1 and $7 \%$ in group $2, p=0.57$ ). No grade $\geq 3$ acute toxicity occurred, and no visual, motor or sensory deficits were recorded during SRT.

During the follow up, RN was suspected in 19 patients (10\%): 10/99 patients (10.1\%) in group 1 and 9/95 (9.5\%) patients in group $2(p=0.9)$. Histological confirmation was obtained in $11 / 19$ patients (58\%). As this procedure was performed to exclude progression, we did not consider it as grade 3 toxicity. The 1-year RN rate was $11.5 \%$ (95\%IC [9.2-13.8]). On univariate analysis, a RPA score $>1$ and the use of concurrent IT were statistically associated with development of $\mathrm{RN}$. On multivariate analysis, the only factor correlated with the occurrence of RN was the use of concurrent IT $(p=0.01)$. The yielding 1-year freedom from $\mathrm{RN}$ was $80 \%$ in patients having received concurrent IT versus $90 \%$ in patients who did not $(p=0.03)$ (Table 5) (figure 3, supplementary materials).

\section{Discussion}

Although series on BMs are heterogeneous in terms of primary tumors, prognosis, treatments and patients clinical characteristics, outcome in our cohort are similar to the one reported in other studies [22, 23].

We found that addition of concurrent IT to SRT was able to increase survival and provide long term control in patients with BMs from solid tumors. In our study, the estimated 1-year LC rates were 96 and $78 \%$ in BM irradiated with versus without concurrent IT $(p=0.022)$. This finding is in line with the recent metaanalyse of Petrelli et al showing that the addition of IT to RT is associated with improved OS $(\mathrm{HR}=0.54,95 \% \mathrm{CI} 0.44-$ 0.67; $p<0.001)$ compared to RT alone in patients treated with BM [24]. The interesting point is that RT given before or concurrently to IT seemed to provide better results than the reverse sequence [25]. Due to the small number of patients (19 received IT before RT, 17 after RT and 9 before and after), we were not able to confirm this hypothesis. One of the explanations for the 
Table 4 Univariate and multivariate analysis for overall survival (OS)

\begin{tabular}{|c|c|c|c|c|c|c|}
\hline \multirow[t]{2}{*}{ Variables } & \multirow[t]{2}{*}{ HR } & \multicolumn{2}{|c|}{ Univariate analysis } & \multirow[t]{2}{*}{ OR } & \multicolumn{2}{|c|}{ Multivariate analysis } \\
\hline & & IC95\% & $p$ & & IC95\% & $p$ \\
\hline Histology (lung vs. others) & 1.52 & $0.97-2.38$ & 0.09 & & & \\
\hline Age (> 55 vs. $\leq 55$ ) & 2.05 & $1.32-3.18$ & 0.004 & 1.04 & $1.09-10.7$ & 0.015 \\
\hline Gender (male vs. female) & 1.23 & $0.80-1.88$ & 0.34 & & & \\
\hline DS-GPA (> 2.5 vs. $\leq 2.5)$ & 0.38 & $0.25-0.58$ & $<0.001$ & 0.8 & $0.56-1.28$ & 0.45 \\
\hline RPA (1 vs. > 1) & 0.61 & $0.41-0.93$ & 0.021 & 0.46 & $0.21-0.97$ & 0.040 \\
\hline Number of BM (1 vs. > 1) & 0.88 & $0.57-1.36$ & 0.58 & & & \\
\hline ECM (yes vs. No) & 1.9 & $1.24-3.10$ & $<0.001$ & 1.70 & $0.77-3.75$ & 0.19 \\
\hline KPS ( $\geq 80 \%$ vs. $<80 \%)$ & 0.29 & $0.18-0.49$ & $<0.001$ & 0.97 & $0.95-0.99$ & 0.040 \\
\hline GTV volume (<2.07 vs. $\geq 2.07)$ & 0.60 & $0.39-0.93$ & 0.015 & 0.93 & $0.85-2.58$ & 0.16 \\
\hline Size (> $10 \mathrm{~mm}$ vs. $<10 \mathrm{~mm}$ ) & 1.32 & $0.83-2.11$ & 0.25 & & & \\
\hline Dose distribution (HG vs. INH) & 1.32 & $0.84-2.08$ & 0.21 & & & \\
\hline ST concurrently (group 1 vs. group 2) & 0.70 & $0.46-1.07$ & 0.09 & & & \\
\hline IT (yes vs. no) & 0.55 & $0.32-0.99$ & 0.08 & & & \\
\hline IT concurrently (yes vs. no IT) & 0.41 & $0.23-0.74$ & 0.02 & 0.22 & $0.08-0.59$ & 0.003 \\
\hline TT (yes vs. no) & 0.79 & $0.51-1.23$ & 0.32 & & & \\
\hline TT concurrently (yes vs. no TKI) & 1.01 & $0.65-1.60$ & 0.95 & & & \\
\hline CT (yes vs. no) & 1.07 & $0.70-1.63$ & 0.73 & & & \\
\hline $\mathrm{CT}$ concurrently (yes vs. no $\mathrm{CT}$ ) & 0.93 & $0.32-1.41$ & 0.72 & & & \\
\hline
\end{tabular}

Abbreviations: DS-GPA Diagnostic-Specific Graded Prognostic Assessment, RPA Recursive partitioning analysis, BM Brain metastases, ECM Extracranial metastases, KPS Karnofsky Performance Status, GTV Gross Tumor Volume, HG Homogeneous, INH Inhomogeneous, ST Systemic Treatment, IT Immunotherapy, $T$ T Targeted therapy, CT Chemotherapy

synergism of that sequence specifically is the RT induced enhancement of cross presentation of antigens by dentritic cells during cancer cell death, triggering the innate immune system to activate tumor-specific $\mathrm{T}$ cells [9]. Moreover, RT administered before systemic treatment may improve the blood-brain barrier permeability, allowing drugs to better penetrate metastases [26].

Moreover, we observed that patients irradiated with a heterogeneous dose developed less distant brain

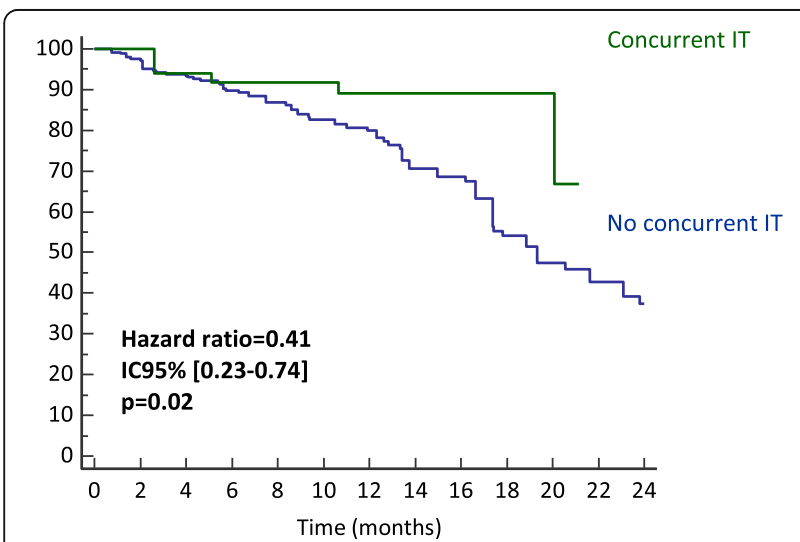

Fig. 1 probability of Overall Survival (OS) depending on the administration of concurrent immunotherapy (IT) metastases than those irradiated with a homogenous dose $(47$ and $71 \%$ at 1 year $(p<0.001)$. This is in line with our previous report, highlighting the importance of SRT planning [27]. An interesting finding here is the trend to a lower intracerebral relapse rate observed in patients receiving concurrent IT compared to those who did not (24\% versus $47 \%$ at 1 year, $p=0.08$ ). This result should however be interpreted with caution given the small number of patients. This observation may reflect the immune-mediated "abscopal effect", defined as tumor regression at sites distant to the irradiated field [28]. Few cases of abscopal effects have been reported in the literature so far, and optimal biological and physical conditions to trigger this immune modulation are unknown. But, case reports on abscopal effects occurring with the combination of anti-CTLA4 and SRT in patients with melanoma are increasing and suggest a clinical benefit in terms of survival $[28,29]$.

The release of antigen for cross presentation depends on the fraction size and total dose of radiation. But, for now, no consensus exists regarding the optimal dose to trigger antigen presentation. Low-dose radiation may better stimulate immune cells and modulate the stromal microenvironment than higher dose [30]. On the opposite, Lee et al showed that a dose of 20 Gy administered in one fraction only induces $\mathrm{T}$ cell proliferation in lymph 
Table 5 Univariate and multivariate analysis for radionecrosis (RN)

\begin{tabular}{|c|c|c|c|c|c|c|}
\hline \multirow[t]{2}{*}{ Variables } & \multirow[t]{2}{*}{ HR } & \multicolumn{2}{|c|}{ Univariate analysis } & \multirow[t]{2}{*}{ OR } & \multicolumn{2}{|c|}{ Multivariate analysis } \\
\hline & & IC95\% & $p$ & & IC95\% & $p$ \\
\hline Histology (lung vs. others) & 1.19 & $0.54-2.65$ & 0.65 & & & \\
\hline Age (> 55 vs. $\leq 55)$ & 0.71 & $0.31-1.63$ & 0.38 & & & \\
\hline Gender (male vs. female) & 0.89 & $0.43-1.89$ & 0.77 & & & \\
\hline DS-GPA (> 2.5 vs. $\leq 2.5)$ & 1.23 & $0.57-2.66$ & 0.57 & & & \\
\hline RPA (1 vs. $>1)$ & 0.40 & $0.20-0.85$ & 0.03 & 0.85 & $0.53-3.15$ & 0.58 \\
\hline Number of BM (1 vs. > 1) & 0.52 & $0.24-1.14$ & 0.07 & & & \\
\hline ECM (yes vs. No) & 0.88 & $0.41-1.89$ & 0.75 & & & \\
\hline KPS (<80\% vs. $\geq 80 \%)$ & 0.99 & $0.42-2.34$ & 0.99 & & & \\
\hline GTV volume (<2.07 vs. $\geq 2.07)$ & 0.71 & $0.33-1.51$ & 0.35 & & & \\
\hline Size (> $10 \mathrm{~mm}$ vs. $<10 \mathrm{~mm})$ & 1.32 & $0.59-2.91$ & 0.51 & & & \\
\hline Dose distribution (HG vs. INH) & 2.01 & $0.96-4.26$ & 0.06 & & & \\
\hline ST concurrently (group 1 vs. group 2) & 2.02 & $0.95-4.32$ & 0.09 & & & \\
\hline IT (yes vs. no) & 2.38 & $0.96-5.89$ & 0.02 & & & \\
\hline IT concurrently (yes vs. no) & 2.23 & $0.90-5.94$ & 0.03 & 3.01 & $1.30-6.94$ & 0.012 \\
\hline TT (yes vs. no) & 1.12 & $0.72-1.95$ & 0.09 & & & \\
\hline TT concurrently (yes vs. no) & 2.66 & $1.19-5.96$ & 0.06 & & & \\
\hline $\mathrm{CT}$ (yes vs. no) & 0.94 & $0.45-1.95$ & 0.87 & & & \\
\hline CT concurrently (yes vs. no) & 0.98 & $0.65-2.06$ & 0.96 & & & \\
\hline Prior WBRT (yes vs. no) & 2.88 & $1.23-6.73$ & 0.07 & & & \\
\hline
\end{tabular}

Abbreviations: DS-GPA Diagnostic-Specific Graded Prognostic Assessment, RPA Recursive partitioning analysis, BM Brain metastases, ECM Extracranial metastases, KPS Karnofsky Performance Status, GTV Gross Tumor Volume, HG Homogeneous, INH Inhomogeneous, ST Systemic Treatment, IT Immunotherapy, TT Targeted therapy, CT Chemotherapy, WBRT Whole brain radiotherapy

nodes whereas this was not observed with the delivery of the same total dose but given in 4 fractions [31].

Radiation-induced brain necrosis is a relatively uncommon but potentially severe adverse event of SRT. Its incidence varies between 2 and 30\% depending on dose prescription and isodose line, dosimetric parameters [27, 32], disease characteristics [33] as well as RN diagnosis criteria [34, 35]. Minniti et al reported RN rates that were significantly different between single- and multiple-fraction SRT (20 and 8\% respectively) [32]. Other authors have reported similar results, with a lower incidence of RN (3 to 11\%) with dose delivery in 3-5 fractions [36, 37]. Although the risk of $\mathrm{RN}$ is known to increase with time, little data are available regarding the risk in longer-term survivors, as survival has generally been poor in the era prior to effective systemic therapy. In a series on $271 \mathrm{BM}$ treated with single-fraction SRT, RN occurred in $25.8 \%$ of treated lesions and, in patients still alive at 2 years, an increase to $34 \%$ was observed [33].

RN could thus be more prevalent in long survivor's patients treated with IT. Furthermore, as RT stimulates the immune response through $\mathrm{T}$-cell activation, it might exacerbate RN [38]. Few studies with limited number of patients have already suggested an increases incidence of $\mathrm{RN}$ with the addition of IT to SRT compared to SRT alone in patients with melanoma [39-41]. But most patients in these reports were treated with the anti-CTLA-4 monoclonal antibody Ipilimumab, and OS rarely exceeded 1 year. In a recent study of Kaidar-Person et al on longer-term surviving patients with metastatic melanoma treated with anti-PD-1 therapy, the incidence of RN was estimated as $18 \%$ at 2 years [40]. We observed a $\mathrm{RN}$ rate of $10 \%$ in the present study, and this rate was higher in patients receiving concomitant IT. This finding has also been reported by Martin et al, who found that receipt of IT was associated with symptomatic RN (HR, 2.56; $p=.004)$ in a cohort of patients with melanoma, nonsmall-cell lung cancer, or renal cell carcinoma, this association being especially strong in patients with melanoma. The association with PD-1 inhibition was however not statistically significant [42].

Our study has inherent bias due to its retrospective nature. First it is heterogeneous, with various tumor types and systemic drugs represented. Many variables were tested including the RPA score, although it can be considered as a confounding factor. Indeed, RPA score combines age, KPS, presence or 
absence of extracranial metastasis, and control of the primary tumor, which were also tested separately. The number of patients who received IT is also limited. Additionally, patients who received concomitant systemic treatment may have been selected based on their performance status or favorable disease presentation or prognosis, and will affect outcomes given the variable time at risk to develop intracranial failure and toxicity. Moreover, oncologists are usually reluctant to interrupt IT in very good responders, this representing a potential selection bias. Systemic treatments could also impact outcome, even without RT. The delay chosen (1 month) to consider systemic treatments as concomitant is also controversial, although largely used in the literature [19, 20, 43]. In a recent meta-analysis published by $\mathrm{Lu}$ et al, it seems that immune checkpoint inhibitor administration within 4 weeks of stereotactic radiosurgery for BMs provides a statistically significant better 12month OS compared to administration outside that time period [43]. Regarding toxicities, only RN was reported as it usually relies on MRI and is therefore relatively easy to collect. But, in the context of systemic treatments, others toxicities are awaited like headaches, cognitive changes, bleeding, or ataxia. These are more difficult to evaluate in the retrospective setting. Finally, the prognostic impact of concurrent IT could be related to the systemic effect of IT, instead of the timing of treatment administration. Given the fact that only 6 patients received not concurrently administered IT, analyzing this subpopulation was not meaningful.

This report provides valuable insight into tolerance and efficacy of combination of IT and SRT. Future clinical trials examining the efficacy and safety of this combination and the optimal schedule of RT in terms of irradiation field, fractionation and dose will hopefully answer these questions. For now, clinicians can rely on the guidelines of the American Society for Radiation Oncology (ASTRO) on combining precision RT with molecular targeting and immunomodulatory agents [44]. Beyond clinical factor and PDL1 expression, other parameters like measure of inflammatory cytokines or tumour-specific T-cells in serum following administration of SRT could act as surrogates for the efficacy of the treatment related immune response, and provide further insight into the appropriate selection of patients.

\section{Conclusion}

SRT delivered concurrently with IT seems to be associated with improved LC, FFDBM and OS as well as with a higher rate of $\mathrm{RN}$.

\section{Supplementary information}

Supplementary information accompanies this paper at https://doi.org/10. 1186/s12885-020-07491-z.

Additional file 1: Figure 1. Probability of local control depending on: (A) administration of concomitant immunotherapy (IT) and (B) metastases volume. Figure 2. Probability of freedom from distant brain metastases (FFDBM) depending on (A) Dose prescription modality and (B) Administration of concurrent immunotherapy (IT). Figure 3. Probability of occurrence of radionecrosis (RN) depending on the administration of concurrent immunotherapy (IT).

\section{Abbreviations}

ALK: Anaplastic lymphoma kinase; ASTRO: American Society for Radiation Oncology; BM: Brain metastases; CT: Computed tomography; CTLA4: Cytotoxic T Iymphocyte-associated antigen 4; DBM: Distant brain metastasis; DS-GPA: Diagnosis-Specific Graded Prognostic Assessment; FDG: Fluorodeoxyglucose; FET: Fluoroethyltyrosine; FF: Flattening-filter; FFDBM: Freedom from distant brain metastases; FLAIR: Fluid-attenuated inversion recovery; HT: Hormonotherapy; IT: Immunotherapy; KPS: Karnosky performance status; LC: Local control; MLC: MultiLeaf Collimator;

MRI: Magnetic resonance imaging; OAR: Organs at risk; OS: Overall survival; PD-1: Programmed death-1; PD-L1: Programmed death ligand-1; PET/ $C T$ : Positron emission tomography/computed tomography; PTV: Planning target volume; RANO-BM: Response Assessment for BM; RN: Radionecrosis; ROC: Receiver operating characteristic; RT: Radiotherapy; SRT: Stereotactic radiotherapy; TKI: Tyrosine kinase inhibitors; TT: Targeted therapy;

VMAT: Volumetric modulated arctherapy

\section{Acknowledgements}

Not applicable.

\section{Authors' contributions}

MG: clinical experiment in stereotactic radiotherapy, study design, statistical analysis, manuscript writing/editing. FL: clinical experiment in stereotactic radiotherapy, study design, statistical analysis, manuscript writing/editing. VB: clinical experiment in stereotactic radiotherapy, statistical analysis, manuscript writing. GD: clinical experiment in stereotactic radiotherapy, manuscript writing. ER: clinical experiment in stereotactic radiotherapy, manuscript writing. GG: Physical experiment in stereotactic radiotherapy, manuscript writing. OP: clinical experiment in stereotactic radiotherapy, manuscript writing. US: clinical experiment in stereotactic radiotherapy, study design, statistical analysis, manuscript writing/editing. All authors have read and approved the manuscript

\section{Funding}

Not applicable.

\section{Availability of data and materials}

The datasets used and/or analysed during the current study are available from the corresponding author on reasonable request.

\section{Ethics approval and consent to participate}

All procedures performed in studies involving human participants were in accordance with the ethical standards of the institutional and/or national research committee and with the 1964 Helsinki declaration and its later amendments or comparable ethical standards.

Informed consent written was obtained from all individual participants included in the study. Institutional Review Board approved this study.

\section{Consent for publication}

Not applicable.

Competing interests

The authors declare that they have no competing interests. 
Received: 24 June 2020 Accepted: 4 October 2020

Published online: 13 October 2020

\section{References}

1. Nayak L, Lee EQ, Wen PY. Epidemiology of brain metastases. Curr Oncol Rep. 2012;14(1):48-54

2. Kocher M, Soffietti R, Abacioglu U, Villa S, Fauchon F, Baumert BG, Fariselli L, Tzuk-Shina T, Kortmann RD, Carrie C, et al. Adjuvant whole-brain radiotherapy versus observation after radiosurgery or surgical resection of one to three cerebral metastases: results of the EORTC 22952-26001 study. J Clin Oncol. 2011;29(2):134-41.

3. Tsao MN, Lloyd N, Wong RKS, et al. Whole brain radiotherapy for the treatment of newly diagnosed multiple brain metastases. Cochrane Database Syst Rev. 2012;(4):CD003869. https://www.ncbi.nlm.nih.gov/pmc/ articles/PMC6457607/.

4. Chang EL, Wefel JS, Hess KR, Allen PK, Lang FF, Kornguth DG, Arbuckle RB, Swint JM, Shiu AS, Maor MH, et al. Neurocognition in patients with brain metastases treated with radiosurgery or radiosurgery plus wholebrain irradiation: a randomised controlled trial. Lancet Oncol. 2009; 10(11):1037-44

5. Brown PD, Jaeckle K, Ballman KV, Farace E, Cerhan JH, Anderson SK, Carrero XW, Barker FG 2nd, Deming R, Burri SH, et al. Effect of radiosurgery alone vs radiosurgery with whole brain radiation therapy on cognitive function in patients with 1 to 3 brain metastases: a randomized clinical trial. JAMA. 2016;316(4):401-9.

6. Yamamoto M, Serizawa T, Shuto T, Akabane A, Higuchi Y, Kawagishi J, Yamanaka K, Sato Y, Jokura H, Yomo S, et al. Stereotactic radiosurgery for patients with multiple brain metastases (JLGK0901): a multi-institutional prospective observational study. Lancet Oncol. 2014;15(4):387-95.

7. Mehta MP, Tsao MN, Whelan TJ, Morris DE, Hayman JA, Flickinger JC, Mills M, Rogers CL, Souhami L. The American Society for Therapeutic Radiology and Oncology (ASTRO) evidence-based review of the role of radiosurgery for brain metastases. Int J Radiat Oncol Biol Phys. 2005;63(1):37-46.

8. Eriksson D, Stigbrand T. Radiation-induced cell death mechanisms. Tumour Biol. 2010;31(4):363-72

9. Demaria S, Golden EB, Formenti SC. Role of local radiation therapy in cancer immunotherapy. JAMA Oncol. 2015;1(9):1325-32.

10. Order SE. The effects of therapeutic irradiation on lymphocytes and immunity. Cancer. 1977;39(2 Suppl):737-43.

11. Robert C, Schachter J, Long GV, Arance A, Grob JJ, Mortier L, Daud A, Carlino MS, McNeil C, Lotem M, et al. Pembrolizumab versus Ipilimumab in Advanced Melanoma. N Engl J Med. 2015;372(26):2521-32.

12. Garon EB, Hellmann MD, Rizvi NA, Carcereny E, Leighl NB, Ahn MJ, Eder JP, Balmanoukian AS, Aggarwal C, Horn L, et al. Five-year overall survival for patients with advanced non-small-cell lung cancer treated with pembrolizumab: results from the phase I KEYNOTE-001 study. J Clin Oncol. 2019;37(28):2518-27.

13. Rini Bl, Plimack ER, Stus V, Gafanov R, Hawkins R, Nosov D, Pouliot $F$, Alekseev B, Soulieres D, Melichar B, et al. Pembrolizumab plus axitinib versus sunitinib for advanced renal-cell carcinoma. N Engl J Med. 2019;380(12): $1116-27$.

14. Twyman-Saint Victor C, Rech AJ, Maity A, Rengan R, Pauken KE, Stelekati E, Benci JL, Xu B, Dada H, Odorizzi PM, et al. Radiation and dual checkpoint blockade activate non-redundant immune mechanisms in cancer. Nature. 2015;520(7547):373-7.

15. Sharabi AB, Lim M, DeWeese TL, Drake CG. Radiation and checkpoint blockade immunotherapy: radiosensitisation and potential mechanisms of synergy. Lancet Oncol. 2015;16(13):e498-509.

16. De Wolf K, Kruse V, Sundahl N, van Gele M, Chevolet I, Speeckaert R, Brochez $L$, Ost $P$. A phase II trial of stereotactic body radiotherapy with concurrent anti-PD1 treatment in metastatic melanoma: evaluation of clinical and immunologic response. J Transl Med. 2017;15(1):21.

17. Ahmed KA, Abuodeh YA, Echevarria MI, Arrington JA, Stallworth DG, Hogue C, Naghavi AO, Kim S, Kim Y, Patel BG, et al. Clinical outcomes of melanoma brain metastases treated with stereotactic radiosurgery and anti-PD-1 therapy, anti-CTLA-4 therapy, BRAF/MEK inhibitors, BRAF inhibitor, or conventional chemotherapy. Ann Oncol. 2016;27(12):2288-94.

18. Mathew M, Tam M, Ott PA, Pavlick AC, Rush SC, Donahue BR, Golfinos JG Parker EC, Huang PP, Narayana A. Ipilimumab in melanoma with limited brain metastases treated with stereotactic radiosurgery. Melanoma Res. 2013;23(3):191-5.
19. Qian JM, Yu JB, Kluger HM, Chiang VL. Timing and type of immune checkpoint therapy affect the early radiographic response of melanoma brain metastases to stereotactic radiosurgery. Cancer. 2016;122(19):3051-8.

20. Kiess AP, Wolchok JD, Barker CA, Postow MA, Tabar V, Huse JT, Chan TA Yamada Y, Beal K. Stereotactic radiosurgery for melanoma brain metastases in patients receiving ipilimumab: safety profile and efficacy of combined treatment. Int J Radiat Oncol Biol Phys. 2015;92(2):368-75.

21. Lin NU, Lee EQ, Aoyama H, Barani IJ, Barboriak DP, Baumert BG, Bendszus M, Brown PD, Camidge DR, Chang SM, et al. Response assessment criteria for brain metastases: proposal from the RANO group. Lancet Oncol. 2015;16(6): e270-8.

22. Sahgal A, Aoyama H, Kocher M, Neupane B, Collette S, Tago M, Shaw P, Beyene J, Chang EL. Phase 3 trials of stereotactic radiosurgery with or without whole-brain radiation therapy for 1 to 4 brain metastases: individual patient data meta-analysis. Int J Radiat Oncol Biol Phys. 2015; 91(4):710-7.

23. Hughes RT, Masters AH, McTyre ER, Farris MK, Chung C, Page BR, Kleinberg LR, Hepel J, Contessa JN, Chiang V, et al. Initial SRS for patients with 5 to 15 brain metastases: results of a multi-institutional experience. Int J Radiat Oncol Biol Phys. 2019;104(5):1091-8.

24. Petrelli F, De Stefani A, Trevisan F, Parati C, Inno A, Merelli B, Ghidini M, Bruschieri L, Vitali E, Cabiddu M, et al. Combination of radiotherapy and immunotherapy for brain metastases: a systematic review and metaanalysis. Crit Rev Oncol Hematol. 2019;144:102830.

25. Pomeranz Krummel DA, Nasti TH, Izar B, Press RH, Xu M, Lowder L, Kallay L, Rupji M, Rosen H, Su J, et al. Impact of sequencing radiation therapy and immune checkpoint inhibitors in the treatment of melanoma brain metastases. Int J Radiat Oncol Biol Phys. 2020;108(1):157-63.

26. Appelboom G, Detappe A, LoPresti M, Kunjachan S, Mitrasinovic S, Goldman S, Chang SD, Tillement O. Stereotactic modulation of blood-brain barrier permeability to enhance drug delivery. Neuro Oncol. 2016;18(12):1601-9.

27. Lucia F, Key S, Dissaux G, Goasduff G, Lucia AS, Ollivier L, Pradier O, Schick U. Inhomogeneous tumor dose distribution provides better local control than homogeneous distribution in stereotactic radiotherapy for brain metastases. Radiother Oncol. 2019;130:132-8.

28. Liu Y, Dong Y, Kong L, Shi F, Zhu H, Yu J. Abscopal effect of radiotherapy combined with immune checkpoint inhibitors. J Hematol Oncol. 2018;11(1): 104.

29. Chicas-Sett R, Morales-Orue I, Rodriguez-Abreu D, Lara-Jimenez P. Combining radiotherapy and ipilimumab induces clinically relevant radiation-induced abscopal effects in metastatic melanoma patients: a systematic review. Clin Transl Radiat Oncol. 2018;9:5-11.

30. Klug F, Prakash H, Huber PE, Seibel T, Bender N, Halama N, Pfirschke C, Voss $\mathrm{RH}$, Timke C, Umansky L, et al. Low-dose irradiation programs macrophage differentiation to an iNOS(+)/M1 phenotype that orchestrates effective T cell immunotherapy. Cancer Cell. 2013;24(5):589-602.

31. Lee $Y$, Auh SL, Wang Y, Burnette B, Wang Y, Meng Y, Beckett M, Sharma R, Chin $R$, Tu $T$, et al. Therapeutic effects of ablative radiation on local tumor require CD8+ T cells: changing strategies for cancer treatment. Blood. 2009; 114(3):589-95

32. Minniti G, Clarke E, Lanzetta G, Osti MF, Trasimeni G, Bozzao A, Romano A, Enrici RM. Stereotactic radiosurgery for brain metastases: analysis of outcome and risk of brain radionecrosis. Radiat Oncol. 2011;6:48.

33. Kohutek ZA, Yamada Y, Chan TA, Brennan CW, Tabar V, Gutin PH, Yang TJ, Rosenblum MK, Ballangrud A, Young RJ, et al. Long-term risk of radionecrosis and imaging changes after stereotactic radiosurgery for brain metastases. J Neuro-Oncol. 2015;125(1):149-56.

34. Blonigen BJ, Steinmetz RD, Levin L, Lamba MA, Warnick RE, Breneman JC. Irradiated volume as a predictor of brain radionecrosis after linear accelerator stereotactic radiosurgery. Int J Radiat Oncol Biol Phys. 2010;77(4): 996-1001.

35. Miller JA, Bennett EE, Xiao R, Kotecha R, Chao ST, Vogelbaum MA, Barnett $\mathrm{GH}$, Angelov L, Murphy ES, Yu JS, et al. Association between radiation necrosis and tumor biology after stereotactic radiosurgery for brain metastasis. Int J Radiat Oncol Biol Phys. 2016;96(5):1060-9.

36. Dore M, Martin S, Delpon G, Clement K, Campion L, Thillays F. Stereotactic radiotherapy following surgery for brain metastasis: predictive factors for local control and radionecrosis. Cancer Radiother. 2017;21(1):4-9.

37. Zhuang H, Zheng Y, Wang J, Chang JY, Wang X, Yuan Z, Wang P. Analysis of risk and predictors of brain radiation necrosis after radiosurgery. Oncotarget. 2016;7(7):7773-9. 
38. Bernstein MB, Krishnan S, Hodge JW, Chang JY. Immunotherapy and stereotactic ablative radiotherapy (ISABR): a curative approach? Nat Rev Clin Oncol. 2016;13(8):516-24.

39. Rahman R, Cortes A, Niemierko A, Oh KS, Flaherty KT, Lawrence DP, Sullivan $R J$, Shih HA. The impact of timing of immunotherapy with cranial irradiation in melanoma patients with brain metastases: intracranial progression, survival and toxicity. J Neuro-Oncol. 2018;138(2):299-306.

40. Kaidar-Person O, Zagar TM, Deal A, Moschos SJ, Ewend MG, Sasaki-Adams D, Lee CB, Collichio FA, Fried D, Marks LB, et al. The incidence of radiation necrosis following stereotactic radiotherapy for melanoma brain metastases: the potential impact of immunotherapy. Anti-Cancer Drugs. 2017;28(6):669-75.

41. Choong ES, Lo S, Drummond M, Fogarty GB, Menzies AM, Guminski A, Shivalingam B, Clarke K, Long GV, Hong AM. Survival of patients with melanoma brain metastasis treated with stereotactic radiosurgery and active systemic drug therapies. Eur J Cancer. 2017:75:169-78.

42. Martin AM, Cagney DN, Catalano PJ, Alexander BM, Redig AJ, Schoenfeld JD, Aizer AA. Immunotherapy and symptomatic radiation necrosis in patients with brain metastases treated with stereotactic radiation. JAMA Oncol. 2018;4(8):1123-4.

43. Lu VM, Goyal A, Rovin RA, Lee A, McDonald KL. Concurrent versus nonconcurrent immune checkpoint inhibition with stereotactic radiosurgery for metastatic brain disease: a systematic review and meta-analysis. J NeuroOncol. 2019:141(1):1-12.

44. Bristow RG, Alexander B, Baumann M, Bratman SV, Brown JM, Camphausen K, Choyke P, Citrin D, Contessa JN, Dicker A, et al. Combining precision radiotherapy with molecular targeting and immunomodulatory agents: a guideline by the American Society for Radiation Oncology. Lancet Oncol. 2018;19(5):e240-51.

\section{Publisher's Note}

Springer Nature remains neutral with regard to jurisdictional claims in published maps and institutional affiliations.

Ready to submit your research? Choose BMC and benefit from:

- fast, convenient online submission

- thorough peer review by experienced researchers in your field

- rapid publication on acceptance

- support for research data, including large and complex data types

- gold Open Access which fosters wider collaboration and increased citations

- maximum visibility for your research: over $100 \mathrm{M}$ website views per year

At $\mathrm{BMC}$, research is always in progress.

Learn more biomedcentral.com/submissions 